\title{
Débat sur l'enseignement de I'histoire : Sur quelques points aveugles
}

\section{Normand Baillargeon}

Au cours de la dernière décennie, dans le contexte de la mise en place d'une ambitieuse et, il faut bien le dire, controversée, réforme de l'éducation, le Québec a été le théâtre d'une vive querelle portant plus spécifiquement sur la réforme de l'enseignement de l'histoire, une querelle si importante qu'une partie des débats qui y ont eu cours a alimenté les pages des quotidiens et rejoint le grand public.

Dans ce débat, le texte d'Éthier, Cardin et Lefrançois (dorénavant : ECL) expose et défend, contre ses détracteurs, quelques-unes des positions cardinales d'une des parties (appelons-la, par commodité, celle des réformistes) contre une autre (appelons-la, toujours par commodité, celle des traditionnalistes). J'y réagirai, pour ma part, non en tant qu'historien ou que spécialiste de la didactique de cette discipline, ce que je ne suis pas, mais en tant que philosophe de l'éducation ayant, pour diverses raisons que j'aurai l'occasion de rappeler ici, pris, dans les débats sur la réforme de l'éducation, une position critique qui m’a amenée à être aussi plutôt critique des transformations que les penseurs tels ECL entendent apporter à l'enseignement de l'histoire.

Mon ambition, modeste, n'est donc pas de rejouer les querelles politiques et idéologiques à multiples facettes qui opposent les uns et les autres dans ce dossier — autour de l'histoire nationale, de l'histoire sociale et de l'histoire politique, notamment — mais de tenter de ramener la conversation vers quelques considérations et problèmes philosophiques et pédagogiques centraux qui me paraissent avoir été occultés.

Mes réactions, ci-après, seront ventilées en trois moments.

Pour commencer, je ferai quelques remarques générales sur le ton de ces débats, que je trouve parfois déplorable, qu'ils aient cours dans les milieux académiques ou sur la place publique. Je voudrais en profiter pour insister sur un de leurs aspects que je trouve particulièrement pernicieux et qui explique sans doute en partie leur ton, à savoir qu'ils présupposent, trop souvent, un faux clivage entre la gauche et la droite, et, ou, entre nationalistes et non-nationalistes (pour employer l'expression la plus neutre possible), qui serait le critère de répartition des positions respectives des protagonistes. 
C'est là, il me semble, une dangereuse simplification qui résulte d'une sorte d'illusion cognitive et qui amène, qui en est victime, à ne pas être en mesure de cerner, non seulement une part importante de ce qui est ici en jeu, mais aussi les positions et argumentaires de certaines des personnes concernées. Ces positions et les argumentaires correspondants, comme on va le voir, se situent en effet, parfois très consciemment, sur un plan pédagogique et philosophique. Il en résulte deux points aveugles auquel je consacrerai mes remarques suivantes.

La première. Je veux rappeler que l'opposition au programme d'histoire des réformistes repose, en partie au moins, pour moi et pour d'autres, sur cette idée que l'approche par compétence et le socioconstructivisme préconisés sont des choix hautement contestables et profondément erronés, voire contre productifs. Cette mienne position peut invoquer en sa faveur à la fois les travaux crédibles de la recherche empirique en éducation et les résultats, eux aussi très crédibles, des sciences cognitives. On trouve en effet, en ces travaux et ces résultats, amplement de quoi alimenter de vives inquiétudes et de grandes suspicions devant l'ambition d'enseigner l'histoire de la manière que les programmes des réformistes le préconisent. Le fait que ces résultats et travaux soient si notoirement absents de leur discours, y compris dans ECL, donne à penser qu'il s'agit là d'un dramatique point aveugle de leur position.

Comme deuxième remarque, je voudrais dire, à mon tour et après tant d'autres, mon inquiétude devant ce que je perçois, moi aussi, comme une instrumentalisation de l'histoire dans le lien que les programmes réformistes tissent entre eux et l'éducation à la citoyenneté. ECL, on le verra, n'a rien fait pour calmer mes inquiétudes.

\section{Un débat d'une singulière virulence}

En disant ce que je m'apprête à dire, je ne veux surtout pas laisser entendre que seule une des parties en présence aurait ces défauts et carences que je veux épingler : mais force est de reconnaître que ce débat sur l'histoire, tant dans les milieux académiques (parfois) que dans les médias (souvent), s'est déroulé dans un climat d'une virulence qui me semble assez rare.

Restons-en à ECL, puisque c'est de ce texte qu'il est question et qu'il ne me semble pas constituer un échantillon qui ne serait pas représentatif du type d'argumentaire qu'on a si souvent rencontré à propos de l'enseignement réformiste de l'histoire. Toutes les citations qui suivent proviennent de ECL et on pourra aisément confirmer, en y retournant, qu'en les rappelant je ne peux être accusé d'en trahir le sens en citant hors contexte.

Les positions des adversaires sont décrites comme s'exprimant à travers des « coups de butoir médiatiques ", comme proposant un " discours de vitupérateurs " lequel est " truffé de demi-vérités ». Ces personnes, qui sont elles aussi des chercheurs et des universitaires, produisent certes des "enquêtes " (mais ce mot est mis entre d'accusateurs guillemets, qui donnent à penser que ces enquêtes n’en sont pas réellement) et des "études" (toujours entre guillemets, pour les mêmes raisons et avec le même effet) «drapant leurs opinions et leurs interprétations de crédibilité scientifique », à la 
" méthodologie discutable au regard des standards de la recherche universitaire ", ce qui n'étonnera pas quand on a décrit nos adversaires comme des "militants engagés dans une cause ", promouvant des " slogans superficiels ", tout ce qui précède étant simplement affirmé et aucunement appuyé par un quelconque argumentaire, comme ce devrait l'être, et encore moins démontré, ce qui témoigne peut-être d'une conception (bien singulière) des "standards de la recherche universitaire ».

Les ad hominem et accusations diverses, non étayées, se multiplient : nos adversaires ont mené une " campagne de désinformation ", nous dit-on; il importe de ne pas être « dupe » de gens dont il faut donc croire qu'ils cherchent à nous tromper; les véritables motivations des personnes épinglées ne sont en outre pas réellement celles qu'elles clament, mais autre chose d'autre : leur lutte est en effet menée "sous couvert » de ce quelque chose, contre des charges " prétendument menées »; elle résulte d'une " réaction d'abord épidermique et peu informée » et peut-être même de causes moins nobles et plus terre à terre, par exemple et pourquoi pas, du fait que ces gens sont « vexés par les rebuffades infligées à leur propre cahier d'exercices ». On évoque même une "théorie du complot contre la nation".

Ces attaques plus ou moins frontales, ces caricatures de la position adverse, sont trop nombreuses pour que ce ne soit troublant et elles invitent à se demander ce qui cause une telle virulence. Je fais l'hypothèse qu'une partie de l'explication tient à des positionnements politiques et idéologiques très polarisés des acteurs. En ce qui concerne les réformistes, et ici même ECL, la perception dominante qu'ils ont d'euxmêmes semble être que leur positionnement est politiquement progressiste (et aussi, mais c'est une autre histoire, épistémologiquement adéquat), tandis que leurs adversaires sont des conservateurs de droite (qui sont aussi, mais c'est également une autre histoire, épistémologiquement déficients). Sitôt mise en place, cette topoï occupe une place surdéterminante dans les débats.

Ce sont donc, nous dit-on d'emblée, " une collation de conservateurs québécois » qui mène cette charge et, sous couvert d'une opposition à un programme, c'est d'un " tramage d'un canevas nationaliste traditionnel " [dont] " ce chœur chante les éloges ». Plus encore, ce mal est planétaire : « [ce] phénomène d'une droite culturelle et identitaire dénonçant des programmes d'études prônant un enseignement épistémologique de l'histoire plutôt que d'être axés sur le patriotisme et la transmission du grand roman national n'a rien de proprement québécois. [...] Dans presque tous les cas, le modèle s'appuie sur une attitude réactionnaire, identitaire et étroitement nationaliste menée par la droite conservatrice des pays concernés ». Bref, selon ECL, " la grille droite-gauche fonctionne bien pour interpréter les différents cas nationaux de cette réaction planétaire."

C'est avec une telle grille en tout cas que se déploie une part non négligeable de l'argumentaire réformiste et par elle qu'on peut, au moins en partie, expliquer le ton et la virulence si communs de ces échanges dont nous avons eu ici un échantillon.

Je soumets que ce virulent débat en cache un autre, le cache parce qu'il l'empêche d'être tenu : ce débat, à la fois pédagogique, scientifique et philosophique, est celui qui porte sur les méthodes pédagogiques préconisées par les réformistes, leurs fondements et leurs éventuels mérites. 


\section{Quelques points aveugles}

Je suis depuis longtemps frappé par le fait que de la même manière que la présence de quelques personnes associées à la " gauche " (dont moi) dans le clan des " traditionnalistes " est pour les réformistes une sorte d'anomalie sans grande importance, la question des méthodes pédagogiques est donnée comme quelque chose d'inessentiel dans toute cette affaire et que leurs adversaires n'auraient invoquée qu'après coup, de manière stratégiquement intéressée. Ici encore, dans ECL, la " critique du constructivisme et de l'approche par compétences » sont données pour être "venues s'ajouter ensuite ". On précise même, et cet argument a été servi à plusieurs reprises, que « lors de la publication du premier programme, personne ne s'inquiéta de ce qu'il se structure autour du développement, par les élèves, de compétences, ni qu'il étudie des sociétés non occidentales, valorisant ainsi la diversité des sociétés, ni même qu'il rejette la mémorisation et l'encyclopédisme ». (ECL)

Ces affirmations ne sont pas entièrement fondées, puisque plusieurs personnes, dont moi, en sont venues à adopter la position critique qui est la leur quant aux programmes réformés d'enseignement de l'histoire d'abord en raison de l'adoption, par lui, des méthodes et des approches pédagogiques qu'ils préconisent.

Ma position est la suivante, pour le dire le plus simplement possible.

Toutes les recherches empiriques crédibles sur l'apprentissage menées en éducation montrent, avec autant de force qu'on peut en attendre en ces matières et pour reprendre une catégorisation proposée par Jeanne Chall, la supériorité de méthodes centrées sur l'enseignant sur celles centrées sur l'élève. Toutes convergent, massivement, vers ce résultat et la récente méta-méta-analyse de la recherche en éducation sur les facteurs d'apprentissage réalisée par John Hattie (2009) le confirme encore, après examen de 800 méta-analyses, synthétisant plus de 50000 recherches ayant porté sur quelque 230 millions de sujets. À propos du constructivisme, justement, entendu mutatis mutandis au sens où les réformistes ici l'entendent, et donc comme théorie de l'apprentissage insistant sur la construction de savoirs et de significations par les élèves à travers leurs activités, Hattie résume ce qu'on peut tirer de cette masse de données en disant que de telles conceptions sont " presque l'exact opposé de la bonne manière d'enseigner et d'apprendre" $(2009,26)$. Ce que tout cela signifie, c'est que les résultats de la recherche crédible, de manière très massivement prévalente, reconnaît comme préférables des méthodes qui vont dans une direction qui n'est pas, globalement, celle que préconisent les approches constructivistes ou socioconstructivistes et par compétence, adoptées tant par la réforme québécoise de l'éducation que par l'enseignement réformé de l'histoire.

De plus, les sciences cognitives confirment la grande plausibilité de ce résultat puisque c'est celui auxquelles elles parviennent elles aussi, par leur voie propre.

Pour le dire le plus simplement possible cette fois encore, compte tenu de la limitation bien connue de notre mémoire de travail par quoi nous accédons au monde et de la nécessité d'en surmonter les limitations par des regroupements que seuls des savoirs permettent, l'enseignement systématique de ces connaissances qui sont 
des préalables au déploiement des capacités cognitives de haut niveau doit précéder celles-ci. Mieux : ces indispensables savoirs préalables doivent être sur-appris pour libérer la mémoire de travail, de sorte qu'en un paradoxe qui en surprendra plus d'un, accéder à ce que les sciences cognitives appellent des savoirs flexibles, qui sont ceux auxquels tout enseignant veut faire accéder ses élèves, suppose, modestement, qu'ils acquièrent d'abord des savoirs inflexibles, la structure de surface des problèmes étant la clé de l'accès à leur structure profonde. Il s'ensuit, selon la même logique, qu'il est risqué, surtout avec des personnes ne possédant pas encore de tels préalables, avec des débutants, d'enseigner selon des méthodes qui demandent le déploiement de ces habiletés cognitives de haut niveau (métacognition, pensée critique, pensée créatrice). Enfin, puisque ces habiletés sont spécifiques à un domaine donné, qu'elles ne se transposent que difficilement étant donné qu'elles présupposent, pour s'exercer, des savoirs dans un domaine donné, rien ne remplace le dur labeur d'acquisition de savoirs préalables avant le déploiement des compétences, lesquelles, pour finir, ne sauraient en aucun cas être transversales si par cela on entend qu'elles puissent s'acquérir une fois pour toute pour ensuite s'exercer en tout contexte (pour un exposé clair et accessible de ces résultats, je suggère : Willingham, 2009).

$\mathrm{Si}$, comme on devrait le faire, on prend au sérieux ce qui précède, on ne peut manquer d'être frappé par la distance qui le sépare de ce que préconise le programme que défend ECL, par exemple en demandant à des débutantes et débutants de " problématiser un phénomène social, [d'] enquêter à son propos à l'aide d'outils propres aux historiens (attitudes, démarche, techniques, concepts, repères culturels et chronologiques, etc.) et [de] prendre en compte les résultats de cette enquête dans sa vie de citoyen ". (ECL) Nous sommes, ici, devant des ambitions que la recherche en éducation et les sciences cognitives auraient dû fortement tempérer, pour dire le moins. J'ignore comment on a pu en venir à promouvoir de telles pratiques si manifestement divergentes des consensus scientifiques, mais je soupçonne que le déplacement de l'expertise en histoire des experts ès contenus disciplinaires aux experts ès sciences de l'éducation, où cette expertise est si peu connue et a tant de mal à être prise en compte, où l'enseignement traditionnel est si souvent caricaturé (ici encore il est présenté comme prônant des " exercices de transcription de mots et de phrases sans recherche de sens ") au profit d'un enseignement donnant un accès immédiat au Saint-Graal des compétences de haut niveau (les « outils propres aux historiens »), que ce déplacement, donc, y est pour quelque chose.

Dans un texte de 2006, Cardin disait clairement que ces programmes avaient de telles ambitions : "enseigner à l'école la pensée historique ", encore une fois en présentant de manière caricaturale ce à quoi on serait condamné si on refuse ces programmes : «faire mémoriser des contenus ». (Cardin, 2006, 63).

Mais ces programmes, disait-il alors aussi, ont une autre grande ambition, sur laquelle ECL revient encore : faire, avec les moyens pédagogiques mis en œuvre, de l'éducation à la citoyenneté et donc « équiper le futur adulte dans son rôle de futur citoyen ». C'est sur cette ambition que je veux avancer mes dernières observations. 


\section{Périls du " citoyennisme"}

Ce volet des nouveaux programmes a suscité de nombreuses critiques et des défenses en aussi grand nombre. D'aucuns ont douté qu'il soit possible de viser simultanément ces deux objectifs; d'autres que les approches préconisées soient les plus susceptibles de permettre d'atteindre celui d'éduquer à la citoyenneté; d'autres encore y ont vu une dangereuse instrumentalisation de l'histoire, tandis qu'on a aussi pu exprimer la crainte d'un recul ou d'une minoration de la place de l'histoire elle-même dans le curriculum. On a même vu un des principaux maîtres d'œuvre des nouveaux programmes, Robert Martineau, prendre ses distances avec cette idée de lier enseignement de l'histoire et éducation à la citoyenneté dans laquelle il voit désormais un "périlleux mariage» (2009).

Comme je l'ai annoncé, ma perspective sur cette question est philosophique et procède d'une conception de l'éducation envisagée dans une perspective libérale (en un sens particulier de ce terme) selon laquelle elle consiste dans la transmission de savoirs retenus pour leur valeur intrinsèque et dans la perspective de leur contribution à la formation d'une personne émancipée, autonome et capable de penser par elle-même. Ces objectifs sont sans doute partagés par la très grande majorité des gens ayant réfléchi à la nature et aux finalités de l'éducation. Pourtant, en leur nom, la philosophie de l'éducation, tout en reconnaissant que la scolarisation puisse entreprendre certaines de ces tâches, invite à une profonde méfiance à l'endroit de toutes ces formes d'éducation à $\mathrm{X}$, qui pullulent aujourd'hui-éducation à la santé, à la sexualité, au loisir, à l'environnement et tutti quanti, et donc aussi à la citoyenneté. C'est qu'en s'éloignant de la transmission de savoirs, on pénètre dans un domaine marqué par la pluralité des positions légitimes et où le risque d'empiéter sur la liberté des sujets est immense. Un concept important permet de penser ces possibles dérives et aide à se prémunir contre elles : celui d'endoctrinement. Je m'étonne donc, je dois le dire, de l'absence de réflexion sérieuse sur tout cela dans la littérature que j'ai lue sur l'enseignement de l'histoire, comme si, en un nouveau point aveugle, ces troublantes et importantes questions n'avaient pas été aperçues.

De plus, à supposer qu'on puisse éviter tous les écueils de l'endoctrinement en enseignant la citoyenneté, concept qui aurait été clairement et assez libéralement défini pour inclure, par exemple, ces adultes qui pensent que nous vivons dans un simulacre de démocratie qu'il faut combattre, j'ai de sérieuses réserves sur le fait que l'histoire, enseignée par les approches ici préconisées, soit une voie pour enseigner cette citoyenneté. C'est pourquoi, ici encore, certaines des affirmations que les méthodes préconisées, centrées sur l'élève et lui enjoignant de "découvrir " par lui-même, non plus des savoirs, mais ce qui est mis en jeu dans cette citoyenneté, laissent pour le moins songeur devant ce qu'on attend de l'élève et ce qu'il est censé construire : "Il n'est pas d'exercice plus formateur des citoyens, écrit ainsi Robert Martineau dans un texte officiel, que de retrouver soi-même les fondements historiques de la citoyenneté pour en apprécier la valeur " (ministère de l'Éducation, 2007, 11).

L'argumentaire déployé par ECL, et en particulier le rappel historique qu'ils déploient pour montrer la permanence d'une dimension citoyenne à l'enseignement de 
l'histoire m'incitent aussi, compte tenu des manifestes violations des esprits qu'on y trouve si souvent, à conclure, différemment d'eux, que c'est là une voie dangereuse, voire fatale et que par souci de préserver les enfants de tout endoctrinement, on ne devrait plus s'y engager. Car nous ne sommes pas plus que nos prédécesseurs à l'abri du risque d'imposer, malgré nous, ce que nous croyons être neutre, juste ou universel et qui ne l'est pas. La modeste transmission de la connaissance du passé, lourde tâche, est ce à quoi le professeur d'histoire devrait s'attacher, ce qui n'interdit pas, cela va sans dire, à l'école d'être un lieu où s'apprend, en la pratiquant, certains aspects de la citoyenneté.

On ne peut savoir d'avance ce que les élèves feront, librement, de ce qu'ils apprennent en histoire (et ailleurs) et des modèles de citoyenneté qu'ils adopteront. C'est là l'inévitable retombée d'une éducation qui forme des esprits libres : ceux-ci penseront et agiront parfois dans des directions qu'on ne soupçonne pas et en certains cas qu'on n'approuve pas.

\section{Références}

Baillargeon, Normand, Contre la réforme. La dérive idéologique du système scolaire québécois (Montréal : PUM, 2009).

Cardin, Jean-François, «Les historiens et le dossier de l'enseignement de l'histoire : chronique d'un passage du centre vers la marge ", Bulletin d'histoire politique, 14, 3.

Chall, J.S., The Academic Achievement Challenge: What Really Works in the Classroom? (New York : The Guilford Press, 2000).

Hattie, J., Visible Learning: A Synthesis of Over 800 Meta-Analyses Relating to Achievement, (New York : Routledge, 2009).

Martineau, Robert, « L'histoire et l'éducation à la citoyenneté... Genèse et fondements d'un périlleux mariage ", Formation et profession, 16, $1: 21-24$.

Ministère de l'Éducation, Programme de formation, Enseignement secondaire. Deuxième cycle. Domaine de l'univers social, Québec, 2007.

Willingham, D. T., Why Don't Students Like School: A Cognitive Scientist Answers Questions About How the Mind Works and What It Means for the Classroom, (San Francisco : JosseyBass, 2009). 